\title{
Molecular gas and star formation in extreme starbursts at low redshift
}

\author{
T. S. Gonçalves ${ }^{1}$, A. Basu-Zych ${ }^{2}$, S. Borthakur ${ }^{3}$, A. Baker ${ }^{4}$, \\ K. Sheth ${ }^{5}$, R. Overzier ${ }^{6}$ and D. C. Martin ${ }^{7}$ \\ ${ }^{1}$ Observatório do Valongo, Universidade Federal do Rio de Janeiro, \\ Ladeira Pedro Antonio 43, Saúde, Rio de Janeiro - RJ, CEP 22240-060 \\ email: tsg@astro.ufrj.br \\ ${ }^{2}$ NASA/GSFC, ${ }^{3}$ Johns Hopkins University, ${ }^{4}$ Rutgers University, \\ ${ }^{5}$ NRAO,${ }^{6}$ UT Austin, ${ }^{7}$ Caltech
}

\begin{abstract}
Lyman Break Analogs (LBAs) are a population of star-forming galaxies at low redshift $(z \sim 0.2)$. These objects present metallicities, morphologies and other physical properties similar to higher redshift Lyman Break Galaxies (LBGs), motivating their detailed study as potential local analogs to high-redshift starburst galaxies (Hoopes et al. 2007, Basu-Zych et al. 2009, Overzier et al. 2010, Overzier et al. 2011).

Our recent integral-field spectroscopy survey of LBAs with Keck/OSIRIS shows that these galaxies have the same nebular gas kinematic properties as high-redshift LBGs. We argue that such kinematic studies are not an appropriate diagnostic to rule out major merger events as the trigger for the observed starburst. Comparison between kinematic analysis and morphological indices from HST imaging disagree with respect to merger identification, with no correlation between the two methods. Artificial redshifting of our data indicates the merger detection rate is even worse at high redshift due to surface brightness dimming and resolution loss. For more details, we refer the reader to Gonçalves et al. (2010).

More recently, we have initiated a program with the CARMA array to detect molecular gas in these galaxies (Gonçalves et al. 2013, in prep.). Our results show that LBAs present strong emission in $\mathrm{CO}(1-0)$, with similar gas fractions to those observed in high-redshift starburst galaxies. Moreover, the objects observed thus far follow the same Schmidt-Kennicutt law as local galaxies, albeit at much higher surface densities, as found in BzK galaxies at $z \sim 2$ (Daddi et al. 2010). This distinguishes our sample from dusty starburst galaxies, such as ULIRGs and SMGs.

The upcoming operations of ALMA will definitely make a great impact on this project. We will be able to observe fainter, lower metallicity objects, and at the same time we will map the $\mathrm{CO}$ emission at sub-kpc resolutions. Once those data become available, we hope the scenario for the physical processes regarding the collapse of the molecular clouds and subsequent star formation will become clearer.
\end{abstract}

Keywords. galaxies: formation — galaxies: ISM — galaxies: high-redshift

\section{References}

Basu-Zych, A. R., Gonçalves, T. S., Overzier, R. et al. 2009, ApJ, 699, L118

Daddi, E., Elbaz, D., Walter, F. et al. 2010, ApJ, 714, L118

Gonçalves, T. S., Basu-Zych, A., Overzier, R. et al. 2010, ApJ, 724, 1373

Overzier, R., Heckman, T., Schiminovich, D., Basu-Zych, A. R., Gonçalves, T. S., Martin, D. C., Rich, R. M. et al. 2010, ApJ, 710, 979

Overzier, R., Heckman, T. M. Wang, J. et al., ApJ, 726, L7 